\title{
Lacunary statistical convergence of double sequences in topological groups
}

Ekrem Savaş*

\section{"Correspondence:}

ekremsavas@yahoo.com

Department of Mathematics,

istanbul Commerce University,

Uskudar, İstanbul, Turkey

\section{第 Springer}

\begin{abstract}
Recently, Patterson and Savaş (Math. Commun. 10:55-61, 2005), defined the lacunary statistical analog for double sequences $x=(x(k, l))$ as follows: A real double sequences $x=(x(k, l))$ is said to be $P$-lacunary statistically convergent to $L$ provided that for each $\varepsilon>0, P$ - $\lim _{r, s} \frac{1}{h_{r s}}\left|\left\{(k, l) \in I_{r, s}:|x(k, l)-L| \geq \varepsilon\right\}\right|=0$. In this case write $s t_{\theta}^{2}-\lim x=L$ or $x(k, l) \rightarrow L\left(s t_{\theta}^{2}\right)$.

In this paper we introduce and study lacunary statistical convergence for double sequences in topological groups and we shall also present some inclusion theorems. MSC: Primary 42B15; secondary 40C05
\end{abstract}

Keywords: double lacunary; double lacunary statistical convergence; topological groups

\section{Introduction}

The notion of statistical convergence, which is an extension of the usual idea of convergence, was introduced by Fast [1] and also independently by Schoenberg [2] for real and complex sequences, but rapid developments were started after the papers of Šalát [3] and Fridy [4]. Nowadays it has become one of the most active area of research in the field of summability. Di Maio and Kočinac [5] introduced the concept of statistical convergence in topological spaces and statistical Cauchy condition in uniform spaces and established the topological nature of this convergence. Statistical convergence has several applications in different fields of mathematics: summability theory, number theory, trigonometric series, probability theory, measure theory, optimization, and approximation theory. Recently a lot of interesting developments have occurred in double statistical convergence and related topics (see [6-9] and [10]).

Before continuing with this paper we present some definitions and preliminaries.

By $X$ we will denote an Abelian topological Hausdorff group, written additively, which satisfies the first axiom of countability. In [11], a sequence $\left(x_{k}\right)$ in $X$ is called to be statistically convergent to an element $L$ of $X$ if, for each neighborhood $U$ of 0 ,

$$
\lim _{n \rightarrow \infty} \frac{1}{n}\left|\left\{k \leq n: x_{k}-L \notin U\right\}\right|=0,
$$

where the vertical bars indicate the number of elements in the enclosed set and is called statistically Cauchy in $X$ if for each neighborhood $U$ of 0 there exists a positive integer

@2014 Savas; licensee Springer. This is an Open Access article distributed under the terms of the Creative Commons Attribution License (http://creativecommons.org/licenses/by/2.0), which permits unrestricted use, distribution, and reproduction in any medium, provided the original work is properly cited. 
$n_{0}(U)$, depending on the neighborhood $U$, such that

$$
\lim _{n \rightarrow \infty} \frac{1}{n}\left|\left\{k \leq n: x_{k}-x_{n_{0}(U)} \notin U\right\}\right|=0 .
$$

The set of all statistically convergent sequences in $X$ is denoted by $s t(X)$ and the set of all statistically Cauchy sequences in $X$ is denoted by $\operatorname{stC}(X)$. It is well known that $\operatorname{st} C(X)=$ $\operatorname{st}(X)$ when $X$ is complete.

By a lacunary sequence, we mean an increasing sequence $\theta=\left(k_{r}\right)$ of positive integers such that $k_{0}=0$ and $h_{r}: k_{r}-k_{r-1} \rightarrow \infty$ as $r \rightarrow \infty$. Throughout this paper, the intervals determined by $\theta$ will be denoted by $I_{r}=\left(k_{r-1}, k_{r}\right]$, and the ratio $\left(k_{r}\right)\left(k_{r-1}\right)^{-1}$ will be abbreviated by $q_{r}$.

In another direction, in [12], a new type of convergence called lacunary statistical convergence was introduced as follows: A sequence $\left(x_{k}\right)$ of real numbers is said to be lacunary statistically convergent to $L$ (or $S_{\theta}$-convergent to $L$ ) if, for any $\varepsilon>0$,

$$
\lim _{r \rightarrow \infty} \frac{1}{h_{r}}\left|\left\{k \in I_{r}:\left|x_{k}-L\right| \geq \varepsilon\right\}\right|=0,
$$

where $|A|$ denotes the cardinality of $A \subset \mathbb{N}$. In [12] the relation between lacunary statistical convergence and statistical convergence was established among other things. In [13], Mursaleen and Mohiuddine extended the idea of lacunary statistical convergence with respect to the intuitionistic fuzzy normed space.

Çakalli [14] defined lacunary statistical convergence in topological groups as follows: A sequence $(x(k))$ is said to be $S_{\theta}$-convergent to $L$ (or lacunary statistically convergent to $L$ ) if, for each neighborhood $U$ of $0, \lim _{r \rightarrow \infty}\left(h_{r}\right)^{-1}\left|\left\{k \in I_{r}: x(k)-L \notin U\right\}\right|=0$. In this case, we write

$$
S_{\theta}-\lim _{k \rightarrow \infty} x(k)=L \quad \text { or } \quad x(k) \rightarrow L\left(S_{\theta}\right)
$$

and define

$$
S_{\theta}(X)=\left\{(x(k)): \text { for some } L, S_{\theta}-\lim _{k \rightarrow \infty} x(k)=L\right\} .
$$

\section{Definitions and notations}

By the convergence of a double sequence we mean the convergence in Pringsheim's sense (see [15]). A double sequence $x=(x(k, l))$ is said to be convergent in Pringsheim's sense if for every $\varepsilon>0$ there exists $N \in \mathbf{N}$ such that $|x(k, l)-L|<\varepsilon$ whenever $k, l \geq N$. $L$ is called the Pringsheim limit of $x$. We shall describe such an $x$ more briefly as ' $P$-convergent'.

A double sequence $x=(x(k, l))$ is said to be Cauchy sequence if for every $\varepsilon>0$ there exists $N \in \mathbf{N}$, where $\mathbf{N}$ is the set of natural numbers such that $|x(p, q)-x(k, l)|<\varepsilon$ for all $p \geq k \geq N$ and $q \geq l \geq N$.

Let $K \subseteq \mathbf{N} \times \mathbf{N}$ be a two-dimensional set of positive integers and let $K_{m, n}$ be the numbers of $(i, j)$ in $K$ such that $i \leq n$ and $j \leq m$. Then the two-dimensional analog of natural case density can be defined as follows: The lower asymptotic density of $K$ is defined as

$$
P-\liminf _{m, n} \frac{K_{m, n}}{m n}=\delta_{2}(K) .
$$


In the case when the sequence $\left\{\frac{K_{m, n}}{m n}\right\}_{m, n=1,1}^{\infty, \infty}$ has a limit we say that $K$ has a natural density and is defined as

$$
P-\lim _{m, n} \frac{K_{m, n}}{m n}=\delta_{2}(K)
$$

For example, let $K=\left\{\left(i^{2}, j^{2}\right): i, j \in \mathbf{N} \times \mathbf{N}\right\}$. Then

$$
\delta_{2}(K)=P-\lim _{m, n} \frac{K_{m, n}}{m n} \leq P-\lim _{m, n} \frac{\sqrt{m} \sqrt{n}}{m n}=0
$$

(i.e., the set $K$ has double natural density zero), while the set $\{(i, 2 j): i, j \in \mathbf{N} \times \mathbf{N}\}$ has double natural density $1 / 2$.

Recently the studies of double sequences have seen rapid growth. The concept of double statistical convergence, for the complex case, was introduced by Mursaleen and Edely [16] and others, while the idea of statistical convergence of single sequences was first studied by Fast [1]. Also the double lacunary statistical convergence was introduced by Patterson and Savaş [17].

Mursaleen and Edely have given the main definition.

Definition 2.1 ([16]) A double sequence $x=(x(k, l))$ is said to be $P$-statistically convergent to $L$ provided that for each $\varepsilon>0$

$$
P-\lim _{m, n} \frac{1}{m n}\{\text { number of }(k, l): k<m \text { and } l<n,|x(k, l)-L| \geq \varepsilon\}=0 .
$$

In this case we write $s t^{2}-\lim _{k, l} x(k, l)=L$ and we denote the set of all statistical convergent double sequences by $s t^{2}$.

It is clear that a convergent double sequence is also $s t^{2}$-convergent but the inverse is not true, in general. Also note that a $s t^{2}$-convergence does need not to be bounded. For example, the sequence $x=\left(x_{k, l}\right)$ defined by

$$
x(k, l)= \begin{cases}k l, & \text { if } k \text { and } l \text { are square } \\ 1, & \text { otherwise }\end{cases}
$$

is $s t^{2}$-convergent. Nevertheless it neither is convergent nor bounded.

It should be noted that in [16], the authors proved the following important theorem.

Theorem 2.2 The following statements are equivalent:

(a) $x$ is statistically convergent to $L$;

(b) $x$ is statistically Cauchy;

(c) there exists a subsequence $y$ of $x$ such that

$$
\lim _{j k} y_{j k}=L
$$

The double sequence $\theta=\left\{\left(k_{r}, l_{s}\right)\right\}$ is called double lacunary if there exist two increasing sequences of integers such that

$$
k_{0}=0, \quad h_{r}=k_{r}-k_{k-1} \rightarrow \infty \quad \text { as } r \rightarrow \infty
$$


and

$$
l_{0}=0, \quad \bar{h}_{s}=l_{s}-l_{s-1} \rightarrow \infty \quad \text { as } s \rightarrow \infty .
$$

Notations: $k_{r, s}=k_{r} l_{s}, h_{r, s}=h_{r} \bar{h}_{s}, \theta$ is determined by $I_{r}=\left\{(k): k_{r-1}<k \leq k_{r}\right\}, I_{s}=\left\{(l): l_{s-1}<\right.$ $\left.l \leq l_{s}\right\}, I_{r, s}=\left\{(k, l): k_{r-1}<k \leq k_{r}\right.$ and $\left.l_{s-1}<l \leq l_{s}\right\}, q_{r}=\frac{k_{r}}{k_{r-1}}, \bar{q}_{s}=\frac{l_{s}}{l_{s-1}}$, and $q_{r, s}=q_{r} \bar{q}_{s}$. We will denote the set of all double lacunary sequences by $\mathbf{N}_{\theta_{r, s}}$.

Let $K \subseteq \mathbf{N} \times \mathbf{N}$ have the double lacunary density $\delta_{2}^{\theta}(K)$ if

$$
P-\lim _{r, s} \frac{1}{h_{r, s}}\left|\left\{(k, l) \in I_{r, s}:(k, l) \in K\right\}\right|
$$

exists.

Example 1 Let $\theta=\left\{\left(2^{r}-1,3^{s}-1\right)\right\}$ and $K=\{(k, 2 l): k, l \in \mathbf{N} \times \mathbf{N}\}$. Then $\delta_{2}^{\theta}(K)=0$. But it is obvious that $\delta_{2}(K)=1 / 2$.

In 2005, Patterson and Savaş [17] studied double lacunary statistically convergence by giving the definition for complex sequences.

Definition 2.3 Let $\theta$ be a double lacunary sequence; the double number sequence $x$ is $s t_{\theta}^{2}$-convergent to $L$ provided that, for every $\varepsilon>0$,

$$
P-\lim _{r, s} \frac{1}{h_{r, s}}\left|\left\{(k, l) \in I_{r, s}:|x(k, l)-L| \geq \varepsilon\right\}\right|=0 .
$$

In this case we write $s t_{\theta}^{2}-\lim x=L$ or $x(k, l) \rightarrow L\left(S_{\theta}^{2}\right)$.

More investigation in this direction and more applications of double lacunary and double sequences can be found in [18-20] and [21].

In this presentation, our goal is to extend a few results known in the literature from ordinary (single) sequences to double sequences in topological groups and to give some important inclusion theorems.

Quite recently, Çakalli and Savaş [22] defined the statistical convergence of double sequences $x=(x(k, l))$ of points in a topological group as follows.

In a topological group $X$, a double sequence $x=(x(k, l))$ is called statistically convergent to a point $L$ of $X$ if for each neighborhood $U$ of 0 the set

$$
\{(k, l), k \leq n \text { and } l \leq m: x(k, l)-L \notin U\}
$$

has double natural density zero. In this case we write $S^{2}-\lim _{k, l} x(k, l)=L$ and we denote the set of all statistically convergent double sequences by $S^{2}(X)$.

Now we are ready to give the definition of double lacunary statistical convergence in topological groups.

Definition 2.4 A sequence $(x(k, l))$ is said to be $S_{\theta}^{2}$-convergent to $L$ (or double lacunary statistically convergent to $L$ ) if, for each neighborhood $U$ of 0 ,

$$
P-\lim _{r, s \rightarrow \infty}\left(h_{r, s}\right)^{-1}\left|\left\{(k, l) \in I_{r, s}: x(k, l)-L \notin U\right\}\right|=0 .
$$


In this case, we write

$$
S_{\theta^{-}}^{2} \lim _{k, l \rightarrow \infty} x(k, l)=L \quad \text { or } \quad x(k, l) \rightarrow L\left(S_{\theta}^{2}\right)
$$

and define

$$
S_{\theta}^{2}(X)=\left\{(x(k, l)): \text { for some } L, S_{\theta^{-}}^{2} \lim _{k, l \rightarrow \infty} x(k, l)=L\right\}
$$

and, in particular,

$$
S_{\theta}^{2}(X)_{0}=\left\{(x(k, l)): S_{\theta^{-}}^{2} \lim _{k, l \rightarrow \infty} x(k, l)=0\right\} .
$$

It is obvious that every double lacunary statistically convergent sequence has only one limit, that is, if a sequence is double lacunary statistically convergent to $L_{1}$ and $L_{2}$ then $L_{1}=L_{2}$.

\section{Inclusion theorems}

In this section, we prove some analogs for double sequences. For single sequences such results have been proved by Çakalli [14].

Theorem 3.1 For any double lacunary sequence $\theta=\left\{\left(k_{r}, l_{s}\right)\right\}, S^{2}(X) \subseteq S_{\theta}^{2}(X)$ if and only if $\liminf q_{r}>1$ and $\liminf \bar{q}_{s}>1$.

Proof Sufficiency: Suppose that $\liminf q_{r}>1$, and $\liminf \bar{q}_{s}>1, \liminf q_{r}=\alpha_{1}$, and $\liminf \bar{q}_{s}=\alpha_{2}$, say. Write $\beta_{1}=\left(\alpha_{1}-1\right) / 2$ and $\beta_{2}=\left(\alpha_{2}-1\right) / 2$. Then there exist two positive integers $r_{0}$ and $s_{0}$ such that $q_{r} \geq 1+\beta_{1}$ for $r \geq r_{0}$ and $\bar{q}_{s} \geq 1+\beta_{2}$ for $s \geq s_{0}$. Thus, for $r \geq r_{0}$, and $s \geq s_{0}$

$$
\begin{aligned}
h_{r, s}\left(k_{r}\right)^{-1}\left(l_{s}\right)^{-1} & =\left(1-\left(k_{r-1}\right)\left(k_{r}\right)^{-1}\right)\left(1-\left(l_{s-1}\right)\left(l_{s}\right)^{-1}\right) \\
& =\left(1-\left(q_{r}\right)^{-1}\right)\left(1-\left(q_{s}\right)^{-1}\right) \\
& \geq\left(1-\left(1+\beta_{1}\right)^{-1}\right)\left(1-\left(1+\beta_{2}\right)^{-1}\right) \\
& =\left(\beta_{1}\left(1+\beta_{1}\right)^{-1}\right)\left(\beta_{2}\left(1+\beta_{2}\right)^{-1}\right) .
\end{aligned}
$$

Take any $(x(k, l)) \in S^{2}(X)$, and $S^{2}-\lim _{(k, l) \rightarrow \infty} x(k, l)=L$, say. We have $S_{\theta}^{2}-\lim _{(k, l) \rightarrow \infty} x(k$, $l)=L$. Let us take any neighborhood $U$ of 0 . Then, for $r \geq r_{0}$ and $s \geq s_{0}$, we get

$$
\begin{aligned}
& \left(k_{r}\right)^{-1}\left(l_{s}\right)^{-1}\left|\left\{k \leq k_{r}, l \leq l_{s}: x(k, l)-L \notin U\right\}\right| \\
& \quad \geq\left(k_{r}\right)^{-1}\left(l_{s}\right)^{-1}\left|\left\{(k, l) \in I_{r, s}: x(k, l)-L \notin U\right\}\right| \\
& \quad=h_{r, s}\left(k_{r}\right)^{-1}\left(l_{s}\right)^{-1}\left(h_{r, s}\right)^{-1}\left|\left\{(k, l) \in I_{r, s}: x(k l)-L \notin U\right\}\right| \\
& \quad \geq \beta_{1}\left(1+\beta_{1}\right)^{-1} \beta_{2}\left(1+\beta_{2}\right)^{-1}\left(h_{r, s}\right)^{-1}\left|\left\{(k, l) \in I_{r, s}: x(k, l)-L \notin U\right\}\right| .
\end{aligned}
$$

Hence $S_{\theta}^{2}-\lim _{(k, l) \rightarrow \infty} x(k, l)=L$.

Necessity: Suppose that $\liminf _{r} q_{r}=1$ and $\liminf _{s} \bar{q}_{s}=1$. Then we can choose a subsequence $\left\{\left(k_{r}(j)\right),\left(l_{s}(i)\right)\right\}$ of the lacunary sequence $\theta_{r, s}=\left\{k_{r}, l_{s}\right\}$ such that

$$
k_{r(j)}\left(k_{r(j)-1}\right)^{-1}<1+j^{-1}, \quad k_{r(j)-1}\left(k_{r(j-1)}\right)^{-1}>j
$$


and

$$
l_{s(i)}\left(l_{s(i)-1}\right)^{-1}<1+i^{-1}, \quad l_{s(i)-1}\left(l_{s(i-1)}\right)^{-1}>i,
$$

where $r(j)>r(j-1)+2$ and $s(i)>s(i-1)+2$. Take an element $x$ of $X$ different from 0 . Define a sequence $(x(k, l))$ by $x(k, l)=x$ if $(k, l) \in\left\{I_{r(j), s(i)}\right\}$ for some $j=1,2, \ldots, m, \ldots, i=1,2, \ldots, n, \ldots$, and $x(k, l)=0$ otherwise. Then $(x(k, l)) \in S^{2}(X)$ (in fact $\left.(x(k, l)) \in S_{0}^{2}(X)\right)$. To prove this take any neighborhood $U$ of 0 . Now we may choose a neighborhood $W$ of 0 such that $W \subset U$ and $x \notin W$. On the other hand, for each $m$ and $n$ we can find two positive numbers $j_{m}$ and $i_{n}$ such that $k_{r\left(j_{m}\right)}<m \leq k_{r\left(j_{m}\right)+1}$ and $l_{s\left(i_{n}\right)}<n \leq l_{s\left(i_{n}\right)+1}$. Therefore

$$
\begin{aligned}
&(m n)^{-1}|\{k \leq m, l \leq n: x(k, l) \notin U\}| \\
& \leq k_{r\left(j_{m}\right)}^{-1} l_{s\left(i_{n}\right)}^{-1}|\{k \leq m, l \leq n: x(k, l) \notin W\}| \\
& \leq k_{r\left(j_{m}\right)}^{-1} l_{s\left(i_{n}\right)}^{-1}\left\{\left|\left\{k \leq k_{r\left(j_{m}\right)}, l \leq l_{s\left(i_{n}\right)}: x(k, l) \notin W\right\}\right|\right. \\
&\left.\quad+\left|\left\{k_{r\left(j_{m}\right)}<k \leq m, l_{s\left(i_{n}\right)}<l \leq n: x(k, l) \notin W\right\}\right|\right\} \\
& \leq k_{r\left(j_{m}\right)}^{-1} l_{s\left(i_{n}\right)}^{-1}\left|\left\{k \leq k_{r\left(j_{m}\right)}, l \leq l_{s\left(i_{n}\right)}: x(k, l) \notin W\right\}\right| \\
&+k_{r\left(j_{m}\right)}^{-1}\left(k_{r\left(j_{m}\right)+1}-k_{r\left(j_{m}\right)}\right), l_{s\left(i_{n}\right)}^{-1}\left(l_{s\left(i_{n}\right)+1}-l_{s\left(i_{n}\right)}\right) \\
&<\left(\left(j_{m}+1\right)^{-1}+1+j_{m}^{-1}-1\right)\left(\left(i_{n}+1\right)^{-1}+1+i_{n}^{-1}-1\right) \\
&<\left(\left(j_{m}+1\right)^{-1}+j_{m}^{-1}\right)\left(\left(i_{n}+1\right)^{-1}+i_{n}^{-1}\right)
\end{aligned}
$$

for each $(m, n)$. Hence $(x(k, l)) \in S_{0}^{2}(X)$. Now let us observe that $(x(k, l)) \notin S_{0}^{2}(X)$. Since $X$ is a Hausdorff space, there exists a symmetric neighborhood $V$ of 0 such that $x \notin V$. Hence

$$
\begin{aligned}
& \lim _{j, i \rightarrow \infty}\left(h_{r(j), s(i)}\right)^{-1}\left|\left\{k_{r(j)-1}<k \leq k_{r(j)}, l_{s(i)-1}<l \leq l_{s(i)}: x(k, l) \notin V\right\}\right| \\
& \quad=\lim _{j, i \rightarrow \infty}\left(h_{r(j)}\right)^{-1}\left(h_{s(i)}\right)^{-1}\left(k_{r(j)}-k_{r(j)-1}\right)\left(l_{s(i)}-l_{s(i)-1}\right) \\
& \quad=\lim _{j, i \rightarrow \infty}\left(h_{r(j)}\right)^{-1} h_{r(j)}\left(h_{s(i)}\right)^{-1}\left(h_{s(i)}\right)=1
\end{aligned}
$$

and

$$
\lim _{\substack{r, s \rightarrow \infty \\ r \neq r(j), s \neq s(i), j=i=1,2, \ldots}} h_{r, s}^{-1}\left|\left\{k_{r-1}<k \leq k_{r}, l_{s-1}<l \leq l_{s}: x(k, l)-x \notin V\right\}\right|=1 \neq 0 .
$$

Therefore neither $x$ nor 0 can be a double lacunary statistical limit of $(x(k, l))$. No other point of $X$ can be a double lacunary statistical limit of the sequence as well. Thus $(x(k, l)) \notin$ $S_{\theta}^{2}(X)$. This completes the proof of this theorem.

Theorem 3.2 For any lacunary sequence $\theta=\left\{\left(k_{r}, l_{s}\right)\right\}, S_{\theta}^{2}(X) \subseteq S^{2}(X)$ if and only if $\limsup _{r} q_{r}<\infty$ and $\lim \sup _{s} \bar{q}_{s}<\infty$.

Proof Sufficiency: If $\limsup q_{r}<\infty$ and $\lim \sup _{s} \bar{q}_{s}<\infty$, there exists an $H>0$ such that $q_{r}<H$ and $\bar{q}_{s}<H$ for all $(r, s)$. Let $(x(k, l)) \in S_{\theta}^{2}(X), S_{\theta}^{2}-\lim _{k, l \rightarrow \infty} x(k, l)=L$, say. Take any neighborhood $U$ of 0 . Let $\varepsilon>0$. Write $N_{r, s}=\left\{(k, l) \in I_{r, s}: x(k, l)-L \notin U\right\}$ by the definition 
of a double lacunary statistical convergence, there are positive integers $r_{0}$ and $s_{0}$ such that $N_{r, s}\left(h_{r, s}\right)^{-1}<\varepsilon$ for all $r>r_{0}$ and $s>s_{0}$. Let $M=\max \left\{N_{r, s}: 1 \leq r \leq r_{0}\right.$ and $\left.1 \leq s \leq s_{0}\right\}$ and let $n$ and $m$ be such that $k_{r-1}<m \leq k_{r}$ and $l_{s-1}<n \leq l_{s}$; hence we have

$$
(m n)^{-1}|\{k \leq m, l \leq n: x(k, l)-L \notin U\}| \leq r_{0} s_{0} M\left(k_{r-1}\right)^{-1}\left(l_{s-1}\right)^{-1}+\varepsilon H^{2} q_{r, s} .
$$

Since $\lim _{r, s \rightarrow \infty} k_{r} l_{s}=\infty$, there exist two positive integers $r_{1} \geq r_{0}$ and $s_{1} \geq s_{0}$ such that

$$
\left(k_{r-1}\right)^{-1}\left(l_{s-1}\right)^{-1}<\left(4 r_{0} s_{0} M / \varepsilon\right)^{-1} \quad \text { for } r>r_{1} \text { and } s>s_{1} .
$$

Thus for $r>r_{1}$ and $s>s_{1}$

$$
(m n)^{-1}|\{k \leq m, l \leq n: x(k, l)-L \notin U\}| \leq \frac{\varepsilon}{2}+\frac{\varepsilon}{2}=\varepsilon .
$$

Finally it follows that $S^{2}-\lim _{k, l \rightarrow \infty} x(k, l)=L$.

Necessity: We shall assume that lim $\sup _{r} q_{r}=\infty$ and lim $\sup _{s} \bar{q}_{s}=\infty$. Take an element $x$ of $X$ different from 0 . Construct two subsequences $\left(k_{r(j)}\right)$ and $\left(l_{s(i)}\right)$ of the lacunary sequence $\theta_{r, s}=\left(k_{r}, l_{s}\right)$ such that $k_{r(j)}>j$ and $l_{s(i)}>i, k_{r(j)}>j+3$, and $l_{s(i)}>i+3$ and define a sequence $(x(k, l))$ by $x(k, l)=x$ if $k_{r(j)-1}<k \leq 2 k_{r(j)-1}$ and $l_{s(i)-1}<l \leq 2 l_{s(i)-1}$ for some $i=j=1,2, \ldots$, and $x(k, l)=0$ otherwise. Let $U$ be a symmetric neighborhood of 0 that does not include $x$. Then, for $j, i>1$,

$$
\begin{aligned}
\left(h_{r(j), s(i)}\right)^{-1}\left|\left\{k \leq k_{r(j)}, l \leq l_{s(i)}: x(k, l) \notin U\right\}\right| & <\left(k_{r(j)-1}\right)\left(l_{s(i)-1}\right)\left(h_{r(j), s(i)}\right)^{-1} \\
& <(j-1)^{-1}(i-1)^{-1} .
\end{aligned}
$$

Hence $(x(k, l)) \in S_{\theta}^{2}(X)$. But $(x(k, l)) \notin S^{2}(X)$. For

$$
\begin{aligned}
& \left(2 k_{r(j)-1}\right)^{-1}\left(2 l_{s(i)-1}\right)^{-1}\left|\left\{k \leq 2 k_{r(j)-1}, l \leq 2 l_{s(i)-1}: x(k, l) \notin U\right\}\right| \\
& \quad=\left(2 k_{r(j)-1}\right)^{-1}\left(2 l_{s(i)-1}\right)^{-1}\left[k_{r(1)-1}+k_{r(2)-1}+\cdots+k_{r(j)-1}\right]\left[l_{s(1)-1}+l_{s(2)-1}+\cdots+l_{s(i)-1}\right]>1 / 4,
\end{aligned}
$$

which implies that $(x(k, l))$ cannot be double statistically convergent. This completes the proof of the theorem.

Corollary 3.3 Let $\theta=\left\{\left(k_{r}, l_{S}\right)\right\}$ be a double lacunary sequence, then $S^{2}(X)=S_{\theta}^{2}(X)$ iff

$$
1<\liminf q_{r} q_{r} \leq \lim \sup _{r} q_{r}<\infty
$$

and

$$
1<\liminf q_{s} \leq \lim \sup _{s} q_{s}<\infty
$$

In Section 2 we mentioned that the $S_{\theta}^{2}$-limit is unique. However, it is possible for a sequence to have different $S_{\theta}^{2}$-limits for different $\theta$ 's. The following theorem shows that this situation cannot occur if $x \in S^{2}(X)$. 
Theorem 3.4 If $(x(k, l))$ belongs to both $S^{2}(X)$ and $S_{\theta}^{2}(X)$, then

$$
S^{2}-\lim _{k, l \rightarrow \infty} x(k, l)=S_{\theta^{-}}^{2} \lim _{k, l \rightarrow \infty} x(k, l) .
$$

Proof Take any $(x(k, l)) \in S^{2}(X) \cap S_{\theta}^{2}(X)$ and $S^{2}-\lim _{k, l \rightarrow \infty} x(k, l)=L_{1}, S_{\theta}^{2}-\lim _{k, l \rightarrow \infty} x(k, l)=$ $L_{2}$, say. Assume that $L_{1} \neq L_{2}$. Since $X$ is a Hausdorff space, there exists a symmetric neighborhood $U$ of 0 such that $L_{1}-L_{2} \notin U$. Then we may choose a symmetric neighborhood $W$ of 0 such that $W+W \subset U$. Then we obtain the following inequality:

$$
\begin{aligned}
& \left(k_{m}\right)^{-1}\left(l_{n}\right)^{-1}\left|\left\{k \leq k_{n}, l \leq l_{m}: z(k, l) \notin U\right\}\right| \\
& \leq\left(k_{m}\right)^{-1}\left(l_{n}\right)^{-1}\left|\left\{k \leq k_{m}, l \leq l_{n}: x(k, l)-L_{1} \notin W\right\}\right| \\
& \quad+\left(k_{m}\right)^{-1}\left(l_{n}\right)^{-1}\left|\left\{k \leq k_{m}, l \leq l_{m}: L_{2}-x(k, l) \notin W\right\}\right|,
\end{aligned}
$$

where $z(k, l)=L_{2}-L_{1}$ for all $k, l \in N \times N$. It follows from this inequality that

$$
\begin{aligned}
1 \leq & \left(k_{m}\right)^{-1}\left(l_{n}\right)^{-1}\left|\left\{k \leq k_{m}, l \leq l_{n}: x(k, l)-L_{1} \notin W\right\}\right| \\
& +\left(k_{m}\right)^{-1}\left(l_{n}\right)^{-1}\left|\left\{k \leq k_{m}, l \leq l_{n}: L_{2}-x(k, l) \notin W\right\}\right| .
\end{aligned}
$$

The second term on the right side of this inequality approaches 0 as $m, n \rightarrow \infty$. To observe this, write

$$
\begin{aligned}
& \left(k_{m}\right)^{-1}\left(l_{n}\right)^{-1}\left|\left\{k \leq k_{m}, l \leq l_{n}: L_{2}-x(k, l) \notin W\right\}\right| \\
& =\left(k_{m}\right)^{-1}\left(l_{n}\right)^{-1}\left|\left\{k, l \in \bigcup_{r, s=1}^{m, n} I_{r, s}: L_{2}-x(k, l) \notin W\right\}\right| \\
& =\left(k_{m}\right)^{-1}\left(l_{n}\right)^{-1} \sum_{r, s=1}^{m, n}\left|\left\{k, l \in I_{r}: L_{2}-x(k, l) \notin W\right\}\right| \\
& =\left(\sum_{r, s=1}^{m, n} h_{r, s}\right)^{-1}\left(\sum_{r, s=1}^{m, n} h_{r, s} t_{r, s}\right),
\end{aligned}
$$

where $t_{r, s}=h_{r, s}^{-1}\left|\left\{k, l \in I_{r, s}: L_{2}-x(k, l) \notin W\right\}\right|$ is a Pringsheim null sequence, since $S_{\theta}^{2}-\lim _{k, l \rightarrow \infty} x(k, l)=L_{2}$. Hence the regular weighted mean transform of $\left(t_{r, s}\right)$ also tends to zero, that is,

$$
P-\lim _{m, n \rightarrow \infty}\left(k_{m}\right)^{-1}\left(l_{n}\right)^{-1}\left|\left\{k \leq k_{m}, l \leq l_{n}: L_{2}-x(k, l) \notin W\right\}\right|=0 .
$$

On the other hand, since $S-\lim _{k, l \rightarrow \infty} x(k, l)=L_{1}$,

$$
P-\lim _{m \rightarrow \infty}\left(k_{m}\right)^{-1}\left(l_{n}\right)^{-1}\left|\left\{k \leq k_{m}, l \leq l_{n}: x(k, l)-L_{1} \notin W\right\}\right|=0 .
$$

By (3.1) and (3.2) it follows that

$$
P \lim _{m, n \rightarrow \infty}\left(k_{m}\right)^{-1}\left(l_{n}\right)^{-1}\left|\left\{k \leq k_{m}, l \leq l_{n}: z(k, l) \notin U\right\}\right|=0 .
$$

This contradiction completes the proof. 
Before presenting the next theorem, let us consider the following definition.

Definition 3.5 Let $\theta=\left(k_{r}, l_{s}\right)$ be a double lacunary sequence; the sequence $(x(k, l))$ is said to be an $S_{\theta}^{2}$-Cauchy sequence if there is a subsequence $\left(x\left(k^{\prime}(r), l^{\prime}(s)\right)\right)$ of $(x(k, l))$ such that $k^{\prime}(r), l^{\prime}(s) \in I_{r, s}$, for each $r, s, \lim _{r, s \rightarrow \infty} x\left(k^{\prime}(r), l^{\prime}(s)\right)=L$ and for each neighborhood $U$ of 0 ,

$$
P-\lim _{r, s \rightarrow \infty}\left(h_{r, s}\right)^{-1}\left|\left\{(k, l) \in I_{r, s}: x(k, l)-x\left(k^{\prime}(r), l^{\prime}(s)\right) \notin U\right\}\right|=0 .
$$

Finally we conclude this paper by presenting the multidimensional analog of Çakalli [14].

Theorem 3.6 A sequence $(x(k, l))$ is $S_{\theta}^{2}$-convergent if and only if it is an $S_{\theta}^{2}$-Cauchy sequence.

Proof Sufficiency: Assume that $(x(k, l))$ is an $S_{\theta}^{2}$-Cauchy sequence. Let $U$ be any neighborhood of 0 . Then we may choose a neighborhood $W$ of 0 such that $W+W \subseteq U$. Therefore

$$
\begin{gathered}
\left(h_{r, s}\right)^{-1}\left|\left\{(k, l) \in I_{r, s}: x(k, l)-L \notin U\right\}\right| \\
\leq\left(h_{r, s}\right)^{-1}\left|\left\{(k, l) \in I_{r, s}: x(k, l)-x\left(k^{\prime}(r), l^{\prime}(s)\right) \notin W\right\}\right| \\
\quad+\left(h_{r, s}\right)^{-1}\left|\left\{(k, l) \in I_{r, s}: x\left(k^{\prime}(r), l^{\prime}(s)\right)-L \notin W\right\}\right| .
\end{gathered}
$$

Since $\lim _{r, s \rightarrow \infty}\left(h_{r, s}\right)^{-1}\left|\left\{(k, l) \in I_{r, s}: x(k, l)-x\left(k^{\prime}(r), l^{\prime}(s)\right) \notin U\right\}\right|=0$, in Pringsheim sense, and $\lim _{r, s \rightarrow \infty} x\left(k^{\prime}(r), l^{\prime}(s)\right)=L$, by the assumption, it follows from the last inequality that $S_{\theta}^{2}-\lim _{k \rightarrow \infty} x(k, l)=L$.

Necessity: Suppose that $S_{\theta}^{2}-\lim _{k, l \rightarrow \infty} x(k, l)=L$. Let $\left(U_{n, m}\right)$ be a nested base of neighborhoods of 0 . Write $K^{(i, j)}=\left\{(k, l) \in N \times N: x(k, l)-L \in U_{i, j}\right\}$ for each $(i, j) \in N \times N$. Thus for each $(i, j)$ we obtain the following: $K^{(i+1, j+1)} \subseteq K^{(i, j)}$ and $\lim _{r, s}\left|K^{(i, j)} \cap I_{r, s}\right|\left(h_{r, s}\right)^{-1}=1$. This implies that there exist $m(1)$ and $n(1)$ such that $r \geq m(1)$ and $s \geq n(1)$ and $\frac{\left|K^{(1,1} \cap I_{r, s}\right|}{h_{r, s}}>0$, that is, $K^{(1,1)} \cap I_{r, s} \neq \varnothing$. We next choose $m(2)>m(1)$ and $n(2)>n(1)$ such that $r>m(2)$ and $s>n(2)$ implies that $K^{(2,2)} \cap I_{r, s} \neq \varnothing$. Thus, for each $(r, s)$ satisfying $m(1) \leq r<m(2)$ and $n(1) \leq s<n(2)$, we can choose $\left(k^{\prime}(r), l^{\prime}(s)\right) \in I_{r, s}$ such that $\left(k^{\prime}(r), l^{\prime}(s)\right) \in K^{(r, s)} \cap I_{r, s}$, that is, $x\left(k^{\prime}(r), l^{\prime}(s)\right)-L \in U_{11}$. In general, we choose $m(p+1)>m(p), n(q+1)>n(q)$ such that $r>m(p+1)$ and $s>n(q+1)$ implies that $I_{r, s} \cap K^{(p+1, q+1)} \neq \varnothing$. Then for all $(r, s)$ satisfying $m(p) \leq r<m(p+1)$ and $n(q) \leq s<n(q+1)$ choose $\left(k^{\prime}(r), l^{\prime}(s)\right) \in I_{r, s} \cap K^{(p, q)}$, that is, $x\left(k^{\prime}(r), l^{\prime}(s)\right)-L \in U_{p, q}$. Hence it follows that $P$ - $\lim _{r, s \rightarrow \infty} x\left(k^{\prime}(r), l^{\prime}(s)\right)=L$. Let $U$ be any neighborhood of 0 . Then we may choose a symmetric neighborhood $W$ of 0 such that $W+W \subseteq U$. Now we write

$$
\begin{aligned}
& \left(h_{r, s}\right)^{-1}\left|\left\{(k, l) \in I_{r, s}: x(k, l)-x\left(k^{\prime}(r), l^{\prime}(s)\right) \notin U\right\}\right| \\
& \leq\left(h_{r, s}\right)^{-1}\left|\left\{(k, l) \in I_{r, s}: x(k, l)-L \notin W\right\}\right| \\
& \quad+\left(h_{r, s}\right)^{-1}\left|\left\{(k, l) \in I_{r, s}: L-x\left(k^{\prime}(r), l^{\prime}(s)\right) \notin W\right\}\right| .
\end{aligned}
$$

Since $S_{\theta}^{2}-\lim _{k, l \rightarrow \infty} x(k, l)=L$ and $P-\lim _{r, s \rightarrow \infty} x\left(k^{\prime}(r), l^{\prime}(s)\right)=L$, we have

$$
\lim _{r, s \rightarrow \infty}\left(h_{r, s}\right)^{-1}\left|\left\{(k, l) \in I_{r, s}: x(k, l)-x\left(k^{\prime}(r), l^{\prime}(s)\right) \notin U\right\}\right|=0,
$$

in Pringsheim's sense. Thus the theorem is proven. 


\section{Competing interests}

The author declares that they have no competing interests.

\section{Acknowledgements}

The author wishes to thank the referees for their valuable suggestions, which have improved the presentation of the paper. This paper was presented during the International Congress in Honour of Professor Ravi P Agarwal at The Auditorium at the Campus of Uludag University, Bursa-Turkey, 23-26 June 2014.

\section{Received: 20 August 2014 Accepted: 7 November 2014 Published: 02 Dec 2014}

\section{References}

1. Fast, H: Sur la convergence statistique. Colloq. Math. 2, 241-244 (1951)

2. Schoenberg, IJ: The integrability methods. Am. Math. Mon. 66, 361-375 (1959)

3. Šalát, T: On statistically convergent sequences of real numbers. Math. Slovaca 30, 139-150 (1980)

4. Fridy, JA: On statistical convergence. Analysis 5, 301-313 (1985)

5. Di Maio, G, Kočinac, LDR: Statistical convergence in topology. Topol. Appl. 156, 28-45 (2008)

6. Mursaleen, M, Çakan, C, Mohiuddine, SA, Savaş, E: Generalized statistical convergence and statistical core of double sequences. Acta Math. Sin. 26(11), 2131-2144 (2010)

7. Savaş, E: On lacunary statistical convergent double sequences of fuzzy numbers. Appl. Math. Lett. 21, 134-141 (2008)

8. Savaş, E: On asymptotically lacunary statistical equivalent sequences of fuzzy numbers. J. Fuzzy Math. 17(3), 527-533 (2009)

9. Savaş, E, Mursaleen, M: On statistically convergent double sequence of fuzzy numbers. Inf. Sci. 162, 183-192 (2004)

10. Savaş, E, Patterson, RF: Double sequence spaces defined by Orlicz functions. Iran. J. Sci. Technol., Trans. A, Sci. 31(2), 183-188 (2007)

11. Çakalli, H: On statistical convergence in topological groups. Pure Appl. Math. Sci. 43(1-2), 27-31 (1996)

12. Fridy, JA, Orhan, C: Lacunary statistical convergence. Pac. J. Math. 160, 43-51 (1993)

13. Mursaleen, M, Mohiuddine, SA: On lacunary statistical convergence with respect to the intuitionistic fuzzy normed space. J. Comput. Appl. Math. 233(2), 142-149 (2009)

14. Çakalli, H: Lacunary statistical convergence in topological groups. Indian J. Pure Appl. Math. 26(2), 113-119 (1995)

15. Pringsheim, A: Zur theorie der zweifach unendlichen Zahlenfolgen. Math. Ann. 53, 289-321 (1900)

16. Mursaleen, M, Edely, OH: Statistical convergence of double sequences. J. Math. Anal. Appl. 288(1), $223-231$ (2003)

17. Patterson, RF, Savaş, E: Lacunary statistical convergence of double sequences. Math. Commun. 10, 55-61 (2005)

18. Savaș, E: On some new double lacunary sequences spaces via Orlicz function. J. Comput. Anal. Appl. 11(3), 423-430 (2009)

19. Savas, E, Patterson, RF: Some double lacunary sequence spaces defined by Orlicz functions. Southeast Asian Bull. Math. 35(1), 103-110 (2011)

20. Savaş, E: Remark on double lacunary statistical convergence of fuzzy numbers. J. Comput. Anal. Appl. 11(1), 64-69 (2009)

21. Savaş, E, Patterson, RF: Double $\sigma$-convergence lacunary statistical sequences. J. Comput. Anal. Appl. 11(4), 610-615 (2009)

22. Çakalli, H, Savaş, E: Statistical convergence of double sequence in topological groups. J. Comput. Anal. Appl. 12(2) $421-426(2010)$

10.1186/1029-242X-2014-480

Cite this article as: Savaş: Lacunary statistical convergence of double sequences in topological groups. Journal of Inequalities and Applications 2014, 2014:480

\section{Submit your manuscript to a SpringerOpen ${ }^{\circ}$ journal and benefit from:}

- Convenient online submission

Rigorous peer review

- Immediate publication on acceptance

Open access: articles freely available online

- High visibility within the field

- Retaining the copyright to your article 